\title{
Resonance of isochronous oscillators
}

\author{
David Rojas
}

Departament d'Enginyeria Informàtica i Matemàtiques, Universitat Rovira i Virgili, Tarragona, Spain

david.rojas@urv.cat

\begin{abstract}
An oscillator such that all motions have the same minimal period is called isochronous. When the isochronous is forced by a time-dependent perturbation with the same natural frequency as the oscillator the phenomenon of resonance can appear. This fact is well understood for the harmonic oscillator and we extend it to the nonlinear scenario.
\end{abstract}

\section{Introduction}

In this communication we present some results from [4] that aim to characterize the class of periodic forcings producing resonances in nonlinear isochronous oscillators.

A well-known fact from physics and mathematics is that the harmonic oscillator with period $2 \pi$ perturbed by a periodic forcing

$$
\ddot{x}+n^{2} x=p(t), n=1,2, \ldots
$$

exhibits resonance whenever the Fourier coefficient

$$
\hat{p}_{n}:=\frac{1}{2 \pi} \int_{0}^{2 \pi} p(t) e^{-i n t} d t
$$

does not vanish. In this context, resonance means that all solutions of the perturbed equation are unbounded. After this example the question that naturally arises is if there exists an equivalent condition for general nonlinear isochronous oscillators. As far as we know this question was first raised by Prof. Roussarie in the Open Problems Session of the II Symposium on Planar Vector Fields (Lleida, 2000).

In this direction, Ortega $[3]$ proved that if the nonlinear isochronous oscillator satisfies a Lipschitz condition then there exist functions $p(t)$ producing resonance. Also Bonheure et all [2] give concrete examples of perturbations. Our contribution in [4] may be interpreted as a nonlinear version of condition $\hat{p}_{n} \neq 0$.

\section{Statement of the results}

Consider the oscillator

$$
\ddot{x}+V^{\prime}(x)=0, x \in \mathbb{R}
$$

where $V \in C^{2}(\mathbb{R})$ is a potential defined on the whole real line satisfying $V(0)=0$, $x V^{\prime}(x)>0$ if $x \neq 0$, and such that all its solutions are $2 \pi$-periodic. The purpose of the following results is to identify the class of $2 \pi$-periodic perturbations $p(t)$ such that all the solutions of the non-autonomous equation

$$
\ddot{x}+V^{\prime}(x)=\varepsilon p(t)
$$


are unbounded for $\varepsilon \neq 0$ small. More precisely, we say that the equation is resonant if every solution $x(t)$ of $(2)$ satisfies

$$
\lim _{|t| \rightarrow+\infty}(|x(t)|+|\dot{x}(t)|)=+\infty .
$$

Let us denote by $\mathcal{C}:=(\mathbb{R} / 2 \pi \mathbb{Z}) \times[0, \infty)$ the cylinder with coordinates $(\theta, r)$. The analogous function that plays the role of the Fourier coefficient $\hat{p}_{n}$ in the nonlinear case is given by the function $\Phi_{p}: \mathcal{C} \rightarrow \mathbb{C}$ defined by

$$
\Phi_{p}(\theta, r):=\frac{1}{2 \pi} \int_{0}^{2 \pi} p(t-\theta) \psi(t, r) d t,
$$

where $\psi(t, r)$ is the complex-valued solution of the variational equation

$$
\ddot{y}+V^{\prime \prime}(\varphi(t, r)) y=0, y(0)=1, \dot{y}(0)=i,
$$

and $\varphi(t, r)$ denotes the solution of system (1) with initial data $x(0)=r$ and $\dot{x}(0)=0$.

[4, Theorem A] states that if $V$ satisfies the previous conditions and $V^{\prime \prime}$ is bounded over the reals then equation (2) is resonant for small $\epsilon \neq 0$ for any $p \in L^{1}(\mathbb{T})$ satisfying the condition

$$
\inf _{\mathcal{C}}\left|\Phi_{p}(\theta, r)\right|>0 .
$$

This result is a sufficient condition for resonance, but in fact condition (3) is not too far from being also necessary. Under the same assumptions on $V$, [4, Proposition 2.2] shows that if $\Phi_{p}$ has a non-degenerate zero $\left(\theta_{*}, r_{*}\right)$ with $r_{*}>0$ then system (2) has a $2 \pi$-periodic solution for small $\epsilon \neq 0$. In particular, resonance is excluded in this situation.

These two results motivate the choice of condition (3) as the nonlinear version of $\hat{p}_{n} \neq 0$ for oscillators defined in the whole real line. Indeed, for the linear oscillator, $V(x)=\frac{1}{2} n^{2} x^{2}, n=1,2, \ldots$ elementary computations lead to the estimates

$$
\frac{1}{2 \pi n}\left|\hat{p}_{n}\right| \leq\left|\Phi_{p}(\theta, r)\right| \leq \frac{1}{2 \pi}\left|\hat{p}_{n}\right|,
$$

which show the equivalence between the condition $\hat{p}_{n} \neq 0$ and (3). However, there are also isochronous oscillators having a singularity. This is the case of the well-known isochronous center

$$
\ddot{x}+\frac{1}{4}\left(x+1-\frac{1}{(x+1)^{3}}\right)=0,
$$

defined for all $x \in(-1,+\infty)$, solved explicitly by Pinney [5]. Bonheure et all [2] considered the perturbed equation

$$
\ddot{x}+\frac{1}{4}\left(x+1-\frac{1}{(x+1)^{3}}\right)=\varepsilon \sin t
$$

and proved that all solutions are unbounded for $\varepsilon \neq 0$ small enough. Our contribution in this scenario is an analogous version of the sufficient condition theorem for resonance. In this case, $[\mathbf{4}$, Theorem $\mathrm{B}]$ proves that if $p \in L^{1}(\mathbb{T})$ satisfies condition (3) then all solutions of the equation

$$
\ddot{x}+\frac{1}{4}\left(x+1-\frac{1}{(x+1)^{3}}\right)=\varepsilon p(t)
$$

are unbounded for $\varepsilon \neq 0$ small enough.

Although this result is stated for Pinney equation, the same proof can be extended to a larger class of potentials $V$ having a singularity. Indeed, Bonheure et all [2] observed 
that usually the existence of a singularity of the potential at $x=a, a<0$, determines the behaviour of $V$ at infinity. This behaviour is precisely the key on the proof of the Theorem. We refer to [4, Appendix] for more details.

The computation of the resonance condition (3) may be difficult in general. In this case, thanks to the contribution of Pinney [5], we are able to compute $\Phi_{p}$ explicitly for the class of linear trigonometric forcings

$$
p(t)=a_{0}+a_{1} \cos t+b_{1} \sin t .
$$

Applying $[\mathbf{4}$, Theorem B] we obtain that equation (5) is resonant if

$$
a_{1}^{2}+b_{1}^{2}>9 a_{0}^{2} .
$$

In particular, we recover the result for (4) in [2].

Motivated by mechanical oscillators, all the perturbations taken into account up to now have been of additive type but in general other kind of perturbations may appear. Inspired by a problem from geometry, Ai, Chou and Wei $[\mathbf{1}]$ studied the equation

$$
\ddot{x}+x=\frac{R(t)}{x^{3}}, x>0
$$

where $R(t)$ is a $T$-periodic function, and proved existence of $T$-periodic solutions when $R$ is a positive $C^{2}$-function and $T<\pi$. The previous equation with $R \equiv 1$ turns out to be isochronous with minimal period $\pi$. This fact suggests that condition $T<\pi$ seems to be sharp due to the presence of resonance. $[4$, Theorem $\mathrm{C}]$ is in the direction of proving this fact, showing that the $\pi$-periodic function

$$
R(t)= \begin{cases}1 & \text { if } t \in\left[0, \frac{\pi}{2}\right), \\ c & \text { if } t \in\left[\frac{\pi}{2}, \pi\right),\end{cases}
$$

with $c>0$ produces that all solutions of (6) are unbounded if $c \neq 1$.

\section{Open problems}

We end this contribution with some related problems that remain unsolved.

First, both the results concerning the identification of the forcings producing resonance for nonlinear isochronous oscillators defined in the whole plane we have presented and the construction of examples by Ortega $[\mathbf{3}]$ require the oscillator to be Lipschitzcontinuous. However, this requirement seems to be a technicality not intrinsically linked to the problem itself but to the proof. We expect that no specific regularity properties of the potential are needed to produce resonance or at least weaker properties than Lipschitz-continuity.

Second, we give a sufficient condition of resonance for the equation (5) perturbed by a linear trigonometric function. Based on the fact that the condition (3) seems also close to be necessary for resonance, it would be interesting to study if equation (5) have periodic orbits for linear trigonometric forcings satisfying $a_{1}^{2}+b_{1}^{2} \leq 9 a_{0}^{2}$.

Third, the example $R(t)$ we have given subscribes the idea that equation (6) exhibits resonance if $R$ is $\pi$-periodic, but it is discontinuous. We think that smooth examples can also be constructed but the approach in [4] do not apply in this situation.

Finally, the results presented deal with nonlinear isochoronous oscillators with one degree of freedom. In more degrees of freedom, the notion of isochronicity is strongly related with the notion of superintegrability, at least in the Hamiltonian framework. 
It would be interesting to relate properly superintegrable Hamiltonian systems with isochronicity and to construct resonance of such systems.

\section{References}

[1] J. Ai, K.S. Chou, J. Wei, Self-similar solutions for the anisotropic affine curve shortening problem, Calc. Var. Partial Differential Equations 13 (2001) 311-337.

[2] D. Bonheure, C. Fabry, D. Smets, Periodic solutions of forced isochronous oscillators at resonance, Discrete Contin. Dyn. Syst. 8 (2002) 907-930.

[3] R. Ortega, Periodic perturbations of an isochronous center, Qualitative Theory of Dynamical Systems 3 (2002) 83-91.

[4] R. Ortega, D. Rojas, Periodic oscillators, isochronous centers and resonance, to appear in Nonlinearity (2018) 1-28. Preprint available at arXiv:1804.03127.

[5] E. Pinney, The nonlinear differential equation $y^{\prime \prime}+p(x) y+c y^{-3}=0$, Proc. Amer. Math. Soc. 1 (1950) 681. 